\title{
Effects of mesh surgery on sexual function in pelvic prolapse and urinary incontinence
}

\author{
Gökmen Sukgen ${ }^{1}$, Adem Altunkol ${ }^{2}$, Ayşe Yiğit ${ }^{3}$ \\ ${ }^{1}$ Sukgen Gynecology and Obstetrics Clinic, Adana, Turkey; ${ }^{2}$ Department of Urology, University of Health \\ Sciences, Adana City Teaching and Research Hospital, Adana, Turkey; ${ }^{3}$ Special Megapol Hospital, \\ Gynecology and Obstetrics Clinic, Istanbul, Turkey
}

\section{ABSTRACT}

Purpose: We aimed to determine pre-operative and post-operative sexual function scores of patients who underwent four-arm polypropylene mesh implantation surgery to treat urinary incontinence and pelvic organ prolapse.

Materials and Methods: A prospective study from January 2011 to November 2015 including patients (n: 72) submitted to surgical mesh implantation (four-arm anterior mesh implant (Betamix POP ${ }^{\circledR}$, Betatech Medical, Turkey) questioned the patients with Female Sexual Function Index evaluation form. The questionnaire was applied to all patients at pre-operative, post-operative 3rd month and post-operative 1st year periods. Results: The mean age of the patients was 47.2 \pm 7.1 years. The mean Body Mass Index $(\mathrm{kg} / \mathrm{m} 2)$ was $28.7 \pm 3.7$. The average of incontinence duration (year) was $4.6 \pm 2.6$ and the average for operation time ( $\mathrm{min}$ ) was $35.7 \pm 2.1$. After the urinary incontinence and pelvic organ prolapse surgery, it was observed that incontinence complaints of patients reduced. Furthermore, there was a positive change in quality of life and sexual function of patients at the post-operative period. There was a statistically significant increase according to Female Sexual Function Index score among all three periods (16\%, 86\% and $100 \%$ respectively, $\mathrm{p}=0.001$ ) and improvement of sexual functions was observed. Conclusions: Transvaginal mesh use in the surgical treatment of pelvic organ prolapse improves quality of life. However, risk factors such as transvaginal mesh usage indication, surgical technique and experience of the surgeon, suitability of the material, the current health status of the patient and postoperative personal care of the patient may affect the success of operations.

\section{ARTICLE INFO}

Gökmen Sukgen

http://orcid.org/0000-0002-1597-2799

Keywords:

Pelvic Organ Prolapse; Urinary

Incontinence; Sexual Behavior

Int Braz J Urol. 2021; 47: 82-9

Submitted for publication:

October 02, 2019

Accepted after revision:

March 16, 2020

Published as Ahead of Print:

May 25, 2020

\section{INTRODUCTION}

Sexuality is a complex process associated with neurological, vascular and endocrine systems (1). Sexuality is one of the main factors affecting the general health status and quality of life of women $(2,3)$. Sexual dysfunction in women is a complex problem that is affected by many bio- logical, psychological and individual factors. Sexual dysfunction in women is a common problem that increases with age and affects approximately $30 \%$ to $50 \%$ of women. However, the prevalence among young women is quite high.

Pelvic organ prolapse (POP) is a type of pelvic floor disorder seen in about one-third of all women. Pelvic organ prolapse refers to the 
outflow or sagging of the bladder, uterus, vagina, small intestine, or rectum of the pelvic floor organs, down the vaginal canal or anus as a result of prolapse. Pelvic organ prolapse, usually in postmenopausal or climacteric period, may be observed sometimes in young women who wish to maintain fertility (1-4). Pelvic floor disorders such as urinary incontinence/pelvic organ prolapse are seen commonly among women at all ages and are not just a long-lasting medical problem but also has negative physiologic, psychological, hygienic, economic and sexual effects which all can be summed up under the term "quality of life" (5). POP incidence is increased with age and POP surgery rates show an increase with time. The aim of POP surgery is to correct the anatomy, symptoms and functions. It is notable that POP surgery increases quality of life (2). POP and urinary incontinence (UI) are common complaints and may present together in the same patient. The most common type of UI is urge - incontinence. SUI is the most frequent type of UI only among middle-aged women which is observed at high intraabdominal pressure conditions such as effort, exertion or sneezing or coughing and defined by International Continence Society (ICS) as urinary incontinence, observed when intravesical pressure exceeds urethra pressure with no increase in detrusor muscle activity (6). It was observed that $63 \%$ of women having SUI have also a descensus while $62 \%$ of women having a descensus have SUI at the same time (7); also, urinary incontinence can be observed in the first three years of menopause at 10\% of women having no complaints previously (8). Vaginal mesh application surgeries came up at POP treatment in addition to conventional pelvic reconstruction methods (9). The first vaginal use of mesh was done by Julian et al. in 1996 and they reported significantly fewer complications and recurrence rates (10). In our country, with increasing industrial pressure in the 2000s, there has been increasing mesh complications in mesh surgery with the contribution of patient incompatible mesh material such as monophilized micropore and insufficient surgical experience. In this context, the warnings of the FDA limiting the use of mesh were unavoidable. However, repairing the fascial defect at the base of the pelvic prolapse with its own fascia, as in conventional surgeries with a high recurrence rate, unfortunately leads to the need for a second surgery due to recurrence. Following studies also supported the place and importance of mesh in the treatment of POP (11, 12). There are variations at the results of pelvic floor surgery's effects on sexual function in literature. Quality of life may improve, stay the same or may become worse after vaginal surgery (13). In our country (Turkey) there are very few studies evaluating the UI and/or POP surgerie's effects on quality of life and sexual functions, which remained under-researched.

In our study, we aimed to compare preoperative and postoperative quality of life and sexual functions of patients having POP and SUI complaints and went through four-arm polypropylene mesh implantation surgery which is accepted as a new method in our clinic.

\section{MATERIALS AND METHODS}

Local ethics committee (2018.01.3111/155) approved this study and informed consent was obtained from all individual participants included in this study. A prospective study from January 2011 to November 2015 including patients in which type 1 polypropylene macropore surgical mesh (Betamix POP $4{ }^{\circledR}$, Betatech Medical, Turkey) implantation were applied with indication of POP and UI and questioned with Female Sexual Function Index (FSFI) evaluation form at outpatient clinic controls with also some information gathered from their computer and file records. Anamneses were taken and routine physical examination, urine culture and office cystometry were done for all patients. This study was conducted at a single center. All operations were performed by a single surgeon (GS).

All urodynamics were performed using 7F transurethral and rectal balloon catheters according to the International Continence Society standards (14). Patients were standardly asked to empty their bladder before this study, and the first step was to measure the postvoid residual (PVR) volume taken from the 7F urethral catheter placed. Filling cystometry was performed with saline. Decreased rate of $30 \mathrm{~mL} / \mathrm{min}$ in cases of severe de- 
trusor overactivity (D0) or known small functional capacity were used in most of the patients included in the present series. A pressure-flow study was obtained at selected times as determined by the clinical scenario (i.e., filling, Valsalva, void/ attempt to void, after voiding). PVR was reassessed after the pressure-flow study. A blind review of all the urodynamic results was done by a urodynamic expert. The following urodynamics data, defined according to the International Continence Society guidelines (14) were collected: cystometric capacity $(\mathrm{mL})$, volume at first sensation of bladder filling $(\mathrm{mL})$, volume at first uninhibited detrusor contraction $(\mathrm{mL})$, the pattern of DO (phasic vs. terminal), maximum flow rate $(\mathrm{Qmax}, \mathrm{mL} / \mathrm{sec})$, detrusor pressure at maximum flow (PdetQmax, cm H20).

The FSFI scoring form was applied to all patients at pre-operation, post-operative 3rd month and post-operative 1st year periods. FSFI was developed by Rosen et al. (15) in the year 2000 and is a test of 19 questions about arousal, sexual desire, orgasm, lubrication, sexual satisfaction and pain. In addition, risk factors that may affect the sociodemographic, obstetric and sexual functions of women are questioned in the questionnaire. Age, age of marriage, education, occupation, education of the spouse, body mass index (BMI) were evaluated as demographic characteristics. The number of children, the type of the last birth, the method of family planning and the frequency of sexual intercourse (weeks) were questioned as obstetric features. Turkish validity and reliability test were carried out for the form by the Turkish Andrology Society in 2003 (16). The scoring is performed by multiplying each question's point with a given ratio and each part is evaluated out of 6 points with a minimum of 2 and a maximum of 36 points in total at the end $(15,16)$. Patients at different POP grades were included. Also, patients with POP had a clinic and cystometric diagnosed SUI. SUI was diagnosed by cystometry and POP grading was done by POP-Q classification clinically. According to POP-Q classification it was observed: Stage 0: No prolapse demonstrated, Stage 1: The most distal portion of the prolapse is greater than $1 \mathrm{~cm}$ above the level of the hymen; Stage 2: The most distal portion of the prolapse is less than $1 \mathrm{~cm}$ above or below the level of the hymen; Stage 3: The most distal portion of the prolapse is greater than $1 \mathrm{~cm}$ below the level of the hymen, but protrudes no further than $2 \mathrm{~cm}$ less than the total vaginal length Stage 4: Complete eversion of the total length of the lower genital tract The distal portion of the prolapse protrudes to at least $2 \mathrm{~cm}$ less than the total vaginal length (17).

\section{SURGERY TECHNIQUE}

Anterior four-arm mesh implant

Surgery was performed under general anesthesia with the patient in the lithotomy position with an indwelling urinary catheter. A linear incision was performed on the anterior vaginal mucosa, about $2.5 \mathrm{~cm}$ below the external urethral opening, and space was dissected until reaching the bladder base. Vesicovaginal ligaments were retracted laterally. The proximal part of the mesh was passed over arcus tendineus fascia pelvis (ATFP) with an obturator fossa guide. The posterior part of the four-arm mesh was passed through the obturator foramen. The anterior arms were placed as a mid-urethral sling as in the intravaginal slingplasty (IVS) procedure, supporting the mid-urethra and bladder. Posterior fringes of the mesh were fixed on cardinal ligaments. About an $8-10 \mathrm{~cm}$ piece of monofilament, inelastic polypropylene tape is attached to the underside of the vaginal apex. Polypropylene sutures are placed into both of the cardinal ligaments and threaded through the lateral edges of the apical sling and tied down, restoring apical support. Posterior mesh arms were fixed at the skin level by performing traction, but the anterior part was not fixed at the mid-urethra and skin level, and tension-free placement was performed.

Prophylactic preoperative antibiotics (cefazolin $1 \mathrm{~g}$, amoxicillin and clavulanic acid $1.2 \mathrm{~g}$ or gentamycin $160 \mathrm{mg}$ ) were administered intravenously. A urinary catheter was removed on the morning of the postoperative day. A vaginal gauze pack (gauze soaked in Betadine iodine) was placed for $12 \mathrm{~h}$. The post-voided residual urine was measured by ultrasonography before each patient was discharged. All the patients received topical intravaginal oestrogen cream treatment for at least 
twelve months following the operation (Ovestin ${ }^{\circledR}$ $1 \mathrm{mg} /$ gram daily).

\section{Statistical analysis}

Patient's pre-operative, post-operative 3rd month and post-operative $1^{\text {st }}$-year results were compared and analyzed. All data were collected by detailed face to face questioning of patient's sociodemographic characteristics, medical and sexual history and risk factors which may cause sexual function disorders. Data were presented as the mean \pm standard deviation. In comparing results, paired student t-test was conducted to compare continuous variables, and chi-square test was conducted to compare categorical variables. The data were analyzed using "SPSS 21 for Windows" software and statistically significance was described by $p$ value $<0.05$.

\section{RESULTS}

Patients with stage 3 and 4 were included in the POP-Q classification. 91 patients were evaluated in total. Patients having a history of pelvic reconstructive surgery (n: 4), not treated infection (n: 3), urinary tract and genital organ lesions (n: 5), any pelvic cancer and/or a history of radiotherapy to the pelvic region (n: 7) were excluded from this study. This study included 72 patients with sexual dysfunction, POP and SUI complaints. The mean of patients' age was $47.2 \pm 7.1, \mathrm{BMI}(\mathrm{kg} /$ $\mathrm{m} 2$ ) was $28.7 \pm 3.7$, incontinence duration (year) was $4.6 \pm 2.6$, operation time (min) was $35.7 \pm 2.1$

Table 1 - Demographic characteristics of patients.

\begin{tabular}{|c|c|c|}
\hline Demographic characteristics & & (n: 72) \\
\hline Age (year) (Mean+std deviation ) & & $47.2 \pm 7.1$ \\
\hline BMI $\left(\mathrm{kg} / \mathrm{m}^{2}\right)$ (Mean+std deviation) & & $28.7 \pm 3.7$ \\
\hline Incontinence Duration (year) (Mean+std deviation) & & $4.6 \pm 2.6$ \\
\hline Operation Time (min) (Mean+std deviation) & & $35.7 \pm 2.1$ \\
\hline Parity(Mean+std deviation) & & $3.4 \pm 1.3$ \\
\hline Marriage Period & & $25.52 \pm 6.45$ \\
\hline Number of children & & $3.12 \pm 0.92$ \\
\hline Sexual intercourse frequency & & $1.02 \pm 0.57$ \\
\hline \multirow{2}{*}{ Education Status $(\mathrm{n}, \%)$} & High school and below & $54(75 \%)$ \\
\hline & University and above & $18(25 \%)$ \\
\hline \multirow{2}{*}{ Education of spouse $(\mathrm{n}, \%)$} & High school and below & $31(43.15 \%)$ \\
\hline & University and above & $41(56.9 \%)$ \\
\hline \multirow{2}{*}{ Prevention method(n,\%) } & Modern method & $58(80.6 \%)$ \\
\hline & Retraction & $14(19.4 \%)$ \\
\hline \multirow[b]{2}{*}{ Type of Birth(n,\%) } & Normal Birth & $49(68.1 \%)$ \\
\hline & Caesarean & $23(31.9 \%)$ \\
\hline
\end{tabular}


(Table-1). According to the distribution of the POP degree, 48 patients had anterior wall, 12 patients had anterior+apical wall and 12 patients had anterior+posterior wall defect.

No early period prolapse as surgery failure was observed in any patient. In none of the patients permanent urinary retention was observed. Only in seven cases, de novo urgency was observed which was successfully corrected with a short period of anticholinergic treatment. In addition, in nine patients, de novo dysuria was observed with no infection in urinary culture but regressed in a short period. In nine patients, forced and intermittent urination was observed with an approximately 100cc post voiding residual volume. In these patients 3-5 days intermittent catheterization was applied, and regression in complaints was observed. In one case, there was an intraoperative urethral injury which was repaired primarily by urologists. In 26 patients postoperative inguinal pain was observed which was treated well with anti-inflammatory and anti-analgesic drugs and physical exercise. In one patient, mesh exposu- re was observed and treatment was obtained with primary repair under local anesthesia and antibiotics . In 11 patients, postoperative dyspareunia was observed. All complications are shown in Table-2.

\section{Ratios}

When the data were described as mean \pm standard deviation, there was a statistically significant increase in FSFI scores when compared preoperatively and postoperative $3^{\text {rd }}$ month, respectively as $21.2 \pm 1.9$ and $24.4 \pm 1.8(\mathrm{p}<0.001)$. When the 1st-year FSFI scores were compared with preoperative scores, there was a statistically significant increase at postoperative first-year scores as well with the level of $27.9 \pm 1.4(p<0.001)$. When the cut-off value for the FSFI score was 26.55, the rates of patients having sexual function disorder preoperatively, postoperative 3rd month and postoperative $1^{\text {st }}$ year were $100 \%, 86 \%$ and $16 \%$ respectively. According to these results, sexual function improved at the end of the first year. The results of the study are shown in Table-3.

Table 2 - Perioperative Complications due to Mesh Implantation.

\begin{tabular}{|c|c|c|}
\hline Complications & Number* & Percent (\%) \\
\hline Permanent Urinary Retention & None & None \\
\hline Transient Urinary Retention & 9 & 12.5 \\
\hline De novo urgency & 7 & 9.7 \\
\hline De novo dysuria & 9 & 12.5 \\
\hline Bladder and Urethral Injury & 1 & 1.3 \\
\hline Pelvic Pain & 26 & 36.1 \\
\hline Mesh Erosion & None & None \\
\hline Mesh Exposure & 1 & 1.3 \\
\hline Major vessel and nerve Injury & None & None \\
\hline Dyspareunia & 11 & 15.2 \\
\hline
\end{tabular}

${ }^{*} n: 72$ 
Table 3 - Preoperative, postoperative 3rd month and 1st year FSFI scores of patients undergoing mesh implantation.

\begin{tabular}{lccc}
\hline FSFI Scores & Pre-operative & $\begin{array}{c}\text { Post-operative } \\
\text { (3th Month) }\end{array}$ & $\begin{array}{c}\text { Post-operative } \\
(1 \text { th Year) }\end{array}$ \\
\hline Mean \pm Std. Deviation & $21.2 \pm 1.9$ & $24.4 \pm 1.8$ & $27.9 \pm 1.4$ \\
Increase in average \% & - & 15.1 & 16 \\
SFD Rate (\%) & 100 & 86 & $* * 0.001$ \\
p-value & - & $*<0.001$ & $* 0.1$ \\
\hline
\end{tabular}

SFD = Sexual Function Disorder; $\mathrm{P}<0.05$ was shown statistically; ${ }^{*}$ Pre-operative- Post-operative (3th Month); ${ }^{* *}$ Pre-operative- Post-operative (1th Year)

\section{DISCUSSION}

POP may lead to altered body image and may have adverse effects on desire, arousal, and orgasm. Furthermore, apart from urinary or prolapse symptoms, as women age, postmenopausal state and chronic diseases may have detrimental effects on sexual functions. It has been shown that older adults desire sexuality when there is a partner and they are in good health state (18). The POP and incontinence surgery improved FSFI scores and sexual functions in approximately $70 \%$ of patients, although some studies that rely on the use of non-condition-specific questionnaires as outcome measures indicate no change (19). We should note that there was an anatomically and functionally significant improvement in patients after the application of four-arm mesh implant.

POP also has a negative impact upon women's emotional health and subjective well-being. POP at a low grade can be successfully treated with conservative management including behavioral modification and pelvic floor muscle exercise. Nevertheless, increasingly POP patients are resorting to surgical intervention to improve their quality of life (20). In this study, the patients reported that the pre-operative anatomical condition caused emotional stress in them and they had gain self confidence after the operation.

POP treatment can be performed surgically or conservatively. There are still controversies among the best surgical technique, which includes; open surgery, laparoscopic or robotic abdominal sacrocolpopexy or anterior and posterior colporrhaphy with or without vaginal hysterectomy. The vaginal repair can be done with or without mesh. The use of mesh has increased due to functional and anatomic improvements when used in appropriate patients (10). In our study, we used four-armed non-absorbable, macroporous monofilament polypropylene synthetic mesh. With the vaginal mesh we have achieved the same functional results with fewer complications.

UI surgery or vaginal dissection may damage vascular and/or neuronal structures which may affect vaginal sense and orgasm (21). It should be kept in mind that although there is normal functioning anatomy, sexual function may be affected by other factors. In our study, we observed a positive effect of UI and POP surgery on the sexual lives of our patients at postoperative 1st year evaluation. Although this overall positive effect on sexual functions in our study shows similar results with other studies, some individual parameters such as sexual desire, orgasm, satisfaction show different results. In our study, there was a significant difference between postoperative $3 \mathrm{rd}$ month and 1st year results and we think this was due to patient's fear of organ damage with intercourse at an early time like three months. When we compared the postoperative 1st year results with preoperative period the significant change was due to sexual dysfunction that arises from UI and POP at the preoperative period. The functional and anatomic correction have an important effect on self-esteem which has a significant positive effect on sexual function disorder, even with a relative evaluation.

Results of the studies evaluating the effects of pelvic floor surgery on sexual functions show differences because of the demographic data differences between groups, differences 
in evaluation methods, stress factors in life, the relationship between partners, age, cultural characteristics and presence of menopause or chronic diseases. The fear of damaging organs after surgery may change the perception of genital health in both partners and may have a negative effect on sexual function. Because of all these reasons, it is hard to say that pelvic floor surgery improves sexual functions in all patients. It is important to provide consultancy about the postoperative sexual function improvement potential and the probable changes in sexual functions to patients who underwent UI and/or POP surgery.

\section{Limitation}

The limitations of our study were a limited number of patients and the short period of post-operative observation.

\section{CONCLUSIONS}

Transvaginal mesh use in the surgical treatment of pelvic organ prolapse improves quality of life. However, risk factors such as transvaginal mesh usage indication, surgical technique and experience of the surgeon, suitability of the material, the current health status of the patient and postoperative personal care of the patient may affect the success of operations. However, randomized controlled trials with more patients are needed.

\section{COMPLIANCE WITH ETHICAL STANDARDS}

Adana City Teaching and Research Hospital Ethical Committee (Adana, Turkey) approved the study protocol. Written informed consent was obtained from all patients included in this study, and this study was conducted in agreement with the Declaration of Helsinki for Medical Research Involving Human Subjects.

\section{CONFLICT OF INTEREST}

\section{REFERENCES}

1. Sivaslioglu AA, Unlubilgin E, Dolen I. A randomized comparison of polypropylene mesh surgery with sitespecific surgery in the treatment of cystocoele. Int Urogynecol J Pelvic Floor Dysfunct. 2008;19:467-71.

2. Citgez S, Demirkesen O, Ozdemir F, Gevher F, Demirdag C, Onal B, et al. Transvaginal repair using acellular collagen biomesh for the treatment of anterior prolapse. Urol J. 2014;11:1271-7.

3. Le Long E, Rebibo JD, Caremel R, Grise P. Efficacy of Pelvisoft ${ }^{\circledR}$ Biomesh for cystocele repair: assessment of long-term results. Int Braz J Urol. 2014;40:828-34.

4. Turgal M, Sivaslioglu A, Yildiz A, Dolen I. Anatomical and functional assessment of anterior colporrhaphy versus polypropylene mesh surgery in cystocele treatment. Eur J Obstet Gynecol Reprod Biol. 2013;170:555-8.

5. Fünfgeld C, Stehle M, Henne B, Kaufhold J, Watermann D, Grebe M, et al. Quality of Life, Sexuality, Anatomical Results and Side-effects of Implantation of an Alloplastic Mesh for Cystocele Correction at Follow-up after 36 Months. Geburtshilfe Frauenheilkd. 2017;77:993-1001.

6. Abrams P, Cardozo L, Fall M, Griffiths D, Rosier P, Ulmsten $\mathrm{U}$, et al. The standardisation of terminology of lower urinary tract function: report from the Standardisation Sub-committee of the International Continence Society. Neurourol Urodyn. 2002;21:167-78.

7. Bai SW, Jeon MJ, Kim JY, Chung KA, Kim SK, Park KH. Relationship between stress urinary incontinence and pelvic organ prolapse. Int Urogynecol J Pelvic Floor Dysfunct. 2002;13:256-60; discussion 260.

8. Herzog AR, Diokno AC, Fultz NH. Urinary incontinence: medical and psychosocial aspects. Annu Rev Gerontol Geriatr. 1989;9:74-119.

9. Moon JW, Chae HD. Vaginal Approaches Using Synthetic Mesh to Treat Pelvic Organ Prolapse. Ann Coloproctol. 2016;32:7-11.

10. Julian TM. The efficacy of Marlex mesh in the repair of severe, recurrent vaginal prolapse of the anterior midvaginal wall. Am J Obstet Gynecol. 1996;175:1472-5.

11. Nguyen JN. The use of grafts for anterior vaginal prolapse repair: pros and ons. Curr Opin Obstet Gynecol. 2008;20:501-5.

12. Hiltunen R, Nieminen K, Takala T, Heiskanen E, Merikari M, Niemi K, et al. Low-weight polypropylene mesh for anterior vaginal wall prolapse: a randomized controlled trial. Obstet Gynecol. 2007;110(2 Pt 2):455-62.

13. Glazener CM, Breeman S, Elders A, Hemming C, Cooper $K G$, Freeman RM, et al. Mesh, graft, or standard repair for 
women having primary transvaginal anterior or posterior compartment prolapse surgery: two parallel-group, multicentre, randomised, controlled trials (PROSPECT). Lancet. 2017;389:381-392.

14. Schäfer W, Abrams $P$, Liao L, Mattiasson A, Pesce F, Spangberg A, et al. Good urodynamic practices: uroflowmetry, filling cystometry, and pressure-flow studies. Neurourol Urodyn. 2002;21:261-74.

15. Rosen R, Brown C, Heiman J, Leiblum S, Meston C, Shabsigh $\mathrm{R}$, et al. The Female Sexual Function Index (FSFI): a multidimensional self-report instrument for the assessment of female sexual function. J Sex Marital Ther. 2000;26:191-208.

16. Çetinel B, Özkan B, Can G. ICIQ-SF TÜRKÇE Versiyon Validasyon (Geçerlilik) Çalışması. Türk Üroloji Dergisi 2004; 30: 332-8.

17. Auwad W, Freeman RM, Swift S. Is the pelvic organ prolapse quantification system (POPQ) being used? A survey of members of the International Continence Society (ICS) and the American Urogynecologic Society (AUGS). Int Urogynecol J Pelvic Floor Dysfunct. 2004:15:324-7.

18. Ugurlucan FG, Evruke I, Yasa C, Dural 0, Yalcin O. Sexual functions and quality of life of women over 50 years with urinary incontinence, lower urinary ract symptoms and/or pelvic organ prolapse. Int J Impot Res. 2019. Online ahead of print.

19. Kammerer-Doak D. Assessment of sexual function in women with pelvic floor dysfunction. Int Urogynecol J Pelvic Floor Dysfunct. 2009;20(Suppl 1):S45-50.

20. Wei D, Wang $P$, Niu $X$, Zhao $X$. Comparison between laparoscopic uterus/sacrocolpopexy and total pelvic floor reconstruction with vaginal mesh for the treatment of pelvic organ prolapse. J Obstet Gynaecol Res. 2019;45:915-922.

21. Onuk Ö. A minimally invasive modified technique for female stress urinary ncontinence: transobturator tape without paraurethral dissection. Wideochir Inne Tech Maloinwazyjne. 2019;14:278-83.

Correspondence address: Gökmen Sukgen, MD Sukgen Gynecology and Obstetrics Clinic, Adana, Turkey Telephone: + 90533 476-9601 E-mail: sukgeng@gmail.com 